\title{
Immirzi ambiguity in the kinematics of quantum general relativity
}

\author{
Luis J. Garay and Guillermo A. Mena Marugán \\ Centro de Física Miguel A. Catalán, I.M.A.F.F., C.S.I.C., Serrano 121, 28006 Madrid, Spain
}

(Received 12 April 2002; published 18 July 2002)

\begin{abstract}
The Immirzi ambiguity arises in loop quantum gravity when geometric operators are represented in terms of different connections that are related by means of an extended Wick transform. We analyze the action of this transform in gravity coupled with matter fields and discuss its analogy with the Wick rotation on which the Thiemann transform between Euclidean and Lorentzian gravity is based. In addition, we prove that the effect of this extended Wick transform is equivalent to a constant scale transformation as far as the symplectic structure and kinematical constraints are concerned. This equivalence is broken in the dynamical evolution. Our results are applied to the discussion of the black hole entropy in the limit of large horizon areas. We first argue that, since the entropy calculation is performed for horizons of fixed constant area, one might in principle choose an Immirzi parameter that depends on this quantity. This would spoil the linearity with the area in the entropy formula. We then show that the Immirzi parameter appears as a constant scaling in all the steps where dynamical information plays a relevant role in the entropy calculation. This fact, together with the kinematical equivalence of the Immirzi ambiguity with a change of scale, is used to preclude the potential nonlinearity of the entropy on physical grounds.
\end{abstract}

DOI: 10.1103/PhysRevD.66.024021

PACS number(s): 04.60.Ds, 04.20.Fy, 04.70.Dy

\section{INTRODUCTION}

The formulation of general relativity in terms of connection variables, introduced by Ashtekar [1,2], constitutes one of the most promising approaches to the quantization of gravity. In the Ashtekar formalism, the gravitational field is described by a complex $S U(2)$ connection and a canonically conjugate, densitized $S U(2)$ soldering form. The shift of emphasis from geometrodynamics to connection dynamics allows the import of techniques employed in the quantization of gauge field theories, providing a common mathematical language for the analysis of quantum gravity and matter. In addition, the expressions of the gravitational constraints in Ashtekar variables are extremely simple, raising renewed hopes for their resolution in the quantum theory.

The price to be paid is that the Ashtekar connection is complex for Lorentzian general relativity. This leads to serious technical and conceptual difficulties, both owing to the lack of a suitable mathematical machinery to deal with the complex $S U(2)$ group and because the real part of the Lorentzian connection turns out to depend on the densitized soldering form, a fact that is incorporated in the quantization program by imposing the so-called reality conditions [2,3].

In order to circumvent these problems, essentially two different avenues have been followed. A possible solution was proposed by Thiemann, who showed that the Lorentzian and Euclidean sectors of Ashtekar gravity can be related by an automorphism on the algebra of functions on phase space $[4,5]$. This automorphism, often called the Thiemann transform, can be regarded as the composition of a Wick transform and a complex constant scale transformation [6,7]. It maps the Lorentzian to the Euclidean constraints and, more importantly, the Ashtekar connection of Lorentzian general relativity to its Euclidean counterpart, which is real. Nonetheless, the complications show up when one tries to implement the Thiemann transform quantum mechanically.

The other possibility was put forward by Barbero. He proved the existence of a (generalized) canonical transformation that converts the Lorentzian complex connection into a real connection [8] (which we will call the Ashtekar-Barbero connection from now on). The only drawback of this change of phase-space variables is that the expression of the Hamiltonian constraint loses its original simplicity. But this relative complication is overwhelmingly compensated for by the availability of the real $S U(2)$ group as the operationally relevant gauge group.

This real connection formalism has been extensively used for the quantization of general relativity, mainly in the framework of loop quantum gravity [9]. Actually, the AshtekarBarbero connection can easily be generalized to a oneparameter family of real connections, all of them related by means of canonical transformations $[8,10]$. The associated parameter is usually called the Immirzi parameter, and we will denote it by $\gamma$. The remarkable point noticed by Immirzi is that the physical predictions of the quantum theory depend on $\gamma$. This is something striking, because the Immirzi parameter designates just equivalent descriptions of the same phase space. From a classical point of view, its value does not affect the physics. Quantum mechanically, however, there exists an ambiguity in $\gamma$ that appears, e.g., as a multiplicative constant in the area spectrum [10].

Recently, a radically different alternative to the AshtekarBarbero formulation has been suggested which is apparently free of the Immirzi ambiguity. This alternative consists in developing a manifestly Lorentz invariant formalism [11]. By retaining the full Lorentz group, one ensures that the choice of quantization scheme does not result in the appearance of anomalies, which could cause the Immirzi ambiguity. In addition, this approach preserves the correct spacetime interpretation of the gravitational variables. In this sense, it is worth commenting that the Ashtekar-Barbero connection has been proved not to transform as the pull-back of a spacetime connection under diffeomorphisms which are normal to the sections of constant time [12]. However, the formalism and 
phase space of this Lorentz invariant approach are much more intricate than those of the original Ashtekar-Barbero theory, and further progress is needed to extract and comprehend its physical predictions.

Since the family of real connections obtained by Immirzi leads to different quantum results, it is clear that the canonical transformations that relate these connections cannot be implemented unitarily [13]. This obstruction for unitarity is not well understood. To shed some light on its origin, the Immirzi ambiguity has been compared with other quantum ambiguities or anomalies. Rovelli and Thiemann [13] have tried to construct a finite dimensional analogue, but their attempt seems to have been unsuccessful [14]. In fact, if one could associate an independent Immirzi ambiguity with every degree of freedom (a finite dimensional system), the ambiguity in general relativity would admit an extension from a constant parameter to two functions of the spatial position [12]. However, this hypothetical extension conflicts with diffeomorphism invariance [15]. On the other hand, Corichi and Krasnov have discussed the possible parallelism between the Immirzi ambiguity and a factor ambiguity that appears in the electric charge of Maxwell theory [16]. But, among standard quantum field theories, the closest similarity is probably found with the $\theta$ ambiguity of Yang-Mill theories [17]. Unlike what happens in that case, however, the Immirzi ambiguity does not arise as a consequence of a multiply connected configuration space $[14,18]$. In this situation, further investigation is required to clarify the roots and implications of the Immirzi ambiguity in quantum gravity.

Some proposed interpretations of this ambiguity have been considered and criticized by Rovelli and Thiemann [13], among them the possibility that the Immirzi parameter amounts to multiplying the classical action by a constant factor. Although both ambiguities are not equivalent, a relation between them should not be discarded. The main reason is that, as pointed out by Rainer [19], the semiclassical predictions of quantum gravity may lead to subtle differences between what we call the Planck length in low-energy physics, $l_{p}$, and what constitutes the fundamental length scale in the quantum theory, $l_{*}$. This fundamental length is determined by the overall factor that multiplies the symplectic structure [20] (or, equivalently, the Poisson brackets). We will return to this issue later in our work.

In connection with the above comments, it has been argued that the Immirzi parameter plays simply the role of a scaling of the Planck length. Since $\gamma$ appears as a global factor in the spectrum of the area operators [10], the Planck length would be multiplied by $\sqrt{\gamma}$. However, the scalar constraint displays a non-homogeneous dependence on $\gamma$ that seems to conflict with this interpretation [17]. One of the aims of the present paper is to discuss the actual relation between the Immirzi ambiguity and a constant scale transformation. We will prove that there indeed exists an equivalence if one restricts all considerations to the kinematics of the Ashtekar-Barbero formulation, i.e., if one disregards dynamics. This analysis will be carried out in gravity with matter fields [3], so that we can also clarify the extent to which the Immirzi ambiguity is or is not affected by the introduction of matter (see the preliminary discussion in Ref. [17]).
We will also discuss the implications of our results for one of the most outstanding predictions of loop quantum gravity: the entropy formula of a quantum black hole. This entropy is calculated assuming a horizon with fixed area $\mathcal{A}$ and adopting a loop quantization with given Immirzi parameter [21-23]. Apparently, therefore, nothing prevents the value of $\gamma$ from depending on $\mathcal{A}$. This would destroy the linear dependence on the area in the deduced entropy $[21,22]$. A way out of this conundrum turns out to be provided by the kinematical equivalence between a change in $\gamma$ and a change of scale. As we will show, this equivalence allows one to regain the Bekenstein-Hawking formula.

The paper is organized as follows. In Sec. II we succinctly describe the Ashtekar formalism for gravity coupled with matter fields. The real Ashtekar-Barbero connection is introduced in Sec. III, where we also revisit the action of constant changes of scale and of some suitable extensions of the Wick and Thiemann transforms. In addition, we analyze the relation between these extended transforms and the canonical transformations introduced by Immirzi. Then, we prove in Sec. IV that the Immirzi ambiguity amounts to a constant scaling as far as the kinematics of general relativity is concerned. The physical consequences of this equivalence are analyzed in Sec. V. In particular, we show that the effect of the Immirzi ambiguity in the formula of the black hole entropy can be absorbed into a change of length scale. Our conclusions are summarized in Sec. VI. Finally, an Appendix is added where we include the expression of the scalar constraint in the presence of matter fields and study how it is affected by the Immirzi ambiguity.

\section{GRAVITY WITH MATTER FIELDS}

Let us briefly review the Hamiltonian formulation of general relativity in the presence of matter fields [2,3]. We will consider a matter content consisting of a massive scalar field $\phi$, massive spin-1/2 fields $\xi_{A}$ and $\bar{\eta}_{A}$, and a Yang-Mills connection $\mathrm{A}_{a}$. All these fields are defined on a certain threemanifold, and we will collectively denote them as $\left\{q^{k}\right\}$. We call $\left\{p_{k}\right\}$ their respective canonical momenta $\left\{\pi_{\phi}, \rho^{A}, \omega^{A}, \mathbb{E}^{a}\right\}$. By canonically conjugate we mean variables whose Poisson bracket is the identity multiplied by $8 \pi l_{*}^{2}$, with $l_{*}=\sqrt{G \hbar}$. Here, $\hbar$ is the Planck constant (i.e., the fundamental quantum of action), $G$ is the true Newton constant that appears in the gravitational action [20], and we have taken $c=1$. Our notation is very similar to that introduced in Ref. [7]. Internal Yang-Mills indices are not displayed, and spatial indices are denoted with lowercase Latin letters from the beginning of the alphabet. Capital Latin letters, on the other hand, designate $S U(2)$ spinors when used as indices. They are raised and lowered with the alternating tensors $\epsilon^{A B}$ and $\epsilon_{A B}$ [2]. Whenever they are not necessary for understanding the formulas, we will also suppress them.

As for the gravitational part of the phase space, it can be described by the canonical pair $\left(a_{a A}{ }^{B}, i \sqrt{2} \sigma^{a}{ }_{A}{ }^{B}\right)$, where $a_{a}$ is the (complex) $S U(2)$ Ashtekar connection and $\sigma^{a}$ is the densitized soldering form [2]. The Ashtekar connection can be written as $a_{a}=\Gamma_{a}-i V_{a}$, where $\Gamma_{a}$ denotes the spin connection compatible with $\sigma^{a}$, and $V_{a}=K_{a}+i C_{a}$, with $K_{a}$ and 
$C_{a}$ being the extrinsic-curvature and fermionic contributions $[2,7]$ :

$$
\begin{aligned}
K_{a} & =\frac{K_{a b} \sigma^{b}}{\sqrt{2} \sigma}, \\
C_{a}^{A B} & =\frac{-i}{4 \sqrt{2}}\left(\sigma_{a}^{A C} y_{C}{ }^{B}+\sigma_{a}^{B C} y_{C}{ }^{A}\right) .
\end{aligned}
$$

In this formula, $K_{a b}$ is the extrinsic curvature, $\sigma_{a}$ is the inverse of $\sigma^{a}, \sigma=\left[\operatorname{det}\left(-\operatorname{tr}\left\{\sigma^{a} \sigma^{b}\right\}\right)\right]^{1 / 4}$, and

$$
y_{A B}=\rho_{A} \xi_{B}+\omega_{A} \bar{\eta}_{B} .
$$

For the action proposed by Ashtekar, Romano and Tate $[2,3]$, the explicit expressions of the kinematical constraints are the following:

$$
\begin{aligned}
\mathrm{G}= & g^{-1} \mathbb{D}_{a} \mathbb{E}^{a}, \\
\mathcal{G}_{A B}= & i \sqrt{2} \breve{\mathcal{D}}_{a} \sigma_{A B}^{a}-y_{(A B)}, \\
\mathcal{V}_{a}= & -i \sqrt{2} \operatorname{tr}\left(\sigma^{b} \mathcal{F}_{a b}\right)-\rho_{A} \breve{\mathcal{D}}_{a} \xi^{A} \\
& -\omega_{A} \breve{\mathcal{D}}_{a} \bar{\eta}^{A}-\pi_{\phi} \partial_{a} \phi-\frac{1}{2} \operatorname{tr}\left(\mathbb{E}^{b} \mathbb{B}_{a b}\right) .
\end{aligned}
$$

Here, $\mathcal{G}$ and $\mathbb{G}$ are the Gauss constraints associated with the Ashtekar and Yang-Mills connections (the latter scaled by the Yang-Mills coupling constant $g^{-1}$ as compared with that in Ref. [2]), and $\mathcal{V}_{a}$ is the vector constraint. The scalar constraint is given in the Appendix.

We will denote as $\left\{\chi_{l}\right\}$ this set of kinematical constraints $\left\{G, \mathcal{G}, \mathcal{V}_{a}\right\}$. In their expressions, $\mathbb{D}_{a}$ is the derivative operator associated with the Yang-Mills connection and $\mathrm{B}_{a b}$ is twice its curvature,

$$
\begin{aligned}
\mathbb{D}_{a} \mathbb{E}^{a} & =\partial_{a} \mathbb{E}^{a}+g\left[\mathrm{~A}_{a}, \mathbb{E}^{a}\right], \\
\mathbb{B}_{a b} & =2\left(\partial_{a} \mathrm{~A}_{b}-\partial_{b} \mathrm{~A}_{a}+g\left[\mathrm{~A}_{a}, \mathrm{~A}_{b}\right]\right) .
\end{aligned}
$$

In addition, $\breve{\mathcal{D}}_{a}$ is the derivative operator associated with the Ashtekar connection and $\mathcal{F}_{a b}$ is its curvature,

$$
\begin{aligned}
\breve{\mathcal{D}}_{a} \xi^{A} & =\partial_{a} \xi^{A}-a_{a}{ }^{A}{ }_{B} \xi^{B}, \\
\mathcal{F}_{a b} & =\partial_{a} a_{b}-\partial_{b} a_{a}+\left[a_{a}, a_{b}\right] .
\end{aligned}
$$

It is worth noting that the only coupling constant on which the kinematical constraints depend is the Yang-Mills one, $g$. As we show in the Appendix, the scalar constraint depends not just on $g$, but also on the fermionic mass $m$ and the scalar field mass $\mu$. Besides, it contains the cosmological constant $\Lambda$, if we allow for a cosmological term in the Hamiltonian. We will generically refer to such parameters as coupling constants and denote their set as $\kappa:=\{g, m, \mu, \Lambda\}$. Finally, the line element can be expressed in terms of the soldering form, the shift vector $N^{a}$ and the densitized lapse function $N$ (with weight equal to -1 ) as

$$
d s^{2}=-\sigma^{2} N^{2} d t^{2}+h_{a b}\left(d x^{a}+N^{a} d t\right)\left(d x^{b}+N^{b} d t\right),
$$

the metric $h_{a b}$ being the inverse of $h^{a b}=-\sigma^{-2} \operatorname{tr}\left(\sigma^{a} \sigma^{b}\right)$.

\section{ASHTEKAR-BARBERO CONNECTIONS}

The Ashtekar connection is not real for two reasons. First, because it is a complex linear combination of the spin connection $\Gamma_{a}$ and the momenta of the soldering forms $V_{a}=K_{a}+i C_{a}$. Second, because these momenta $V_{a}$ are genuinely complex when fermions are present $[2,7]$. With the aim of addressing the former of these issues, we introduce the connections

$$
\gamma_{a_{a}}=\Gamma_{a}-\gamma V_{a}
$$

where $\gamma>0$ is the Immirzi parameter. Since our definition reproduces that of Ref. [8] in the absence of matter, we will call $\gamma_{a}$ the Ashtekar-Barbero connections. It should however be clear that such connections are not real when fermions are allowed. The canonically conjugate momenta of $-\sqrt{2} \gamma a_{a}$ are ${ }^{\gamma} \sigma^{a}=\gamma^{-1} \sigma^{a}$.

Formulations with different values of the Immirzi parameter $\gamma$ are related by an extended Wick transform $R_{\gamma}[7,24]$ such that

$$
\begin{aligned}
& R_{\gamma^{\circ}}{ }^{1} a_{a}=\gamma^{\gamma} a_{a}, \quad R_{\gamma^{\circ}} q^{k}=q^{k}, \\
& R_{\gamma^{\circ}} \sigma^{a}=\sigma^{a}, \quad R_{\gamma^{\circ}} p_{k}=\gamma p_{k} .
\end{aligned}
$$

Note that the action on the gravitational connection can be rephrased as $R_{\gamma} \circ V_{a}=\gamma V_{a}$. Similarly to the interpretation accepted for the case of the (inverse) Wick rotation, where $\gamma$ would be equal to $-i$, the simultaneous multiplication by $\gamma$ of $V_{a}$ and all the momenta of the matter fields can be regarded as the consequence of a scaling of the lapse [7]. Consequently, the action of $R_{\gamma}$ on phase space is supplemented with the following transformation laws on the lapse and shift [25]:

$$
R_{\gamma}^{\circ} N=\gamma^{-1} N, \quad R_{\gamma}^{\circ} N^{a}=N^{a} .
$$

In addition, it seems natural to admit that the transform does not affect the coupling constants, $R_{\gamma}{ }^{\circ} \kappa=\kappa$.

This extended Wick transform does not preserve the symplectic structure. However, as in the case that leads to the Thiemann transform [7], one can complement the extended Wick transform with a constant scale transformation and construct a symplectomorphism that relates formulations with different values of $\gamma$. To show how this can be done, let us first introduce the constant scale transformations:

$$
C_{\beta^{\circ}} X=\beta^{D(X)} X .
$$

Here, $D(X)$ is the dimension of $X$ (a generic field or parameter). Adopting the convention that the dimensionality of the line element is carried by the metric and not by the coordinates, it is possible to show that [7]

$$
D\left({ }^{1} a_{a}\right)=0, \quad D\left(q^{k}\right)=(-1)^{2 s_{k}} s_{k},
$$




$$
\begin{array}{ll}
D\left(\sigma^{a}\right)=2, & D\left(p_{k}\right)=2-(-1)^{2 s_{k} s_{k},} \\
D(N)=-2, & D\left(N^{a}\right)=0, \\
D(\Lambda)=-2, & D(m)=D(\mu)=D(g)=-1 .
\end{array}
$$

In these formulas, $s_{k}$ denotes the spin of the field $q^{k}$.

We have now all the ingredients necessary to construct the extended Thiemann transform $T_{\gamma}$ :

$$
T_{\gamma}:=C_{\gamma^{-1 / 2}} \circ R_{\gamma}
$$

Its action on phase space is

$$
\begin{aligned}
& \gamma_{a_{a}}:=T_{\gamma^{\circ}} a_{a}=\Gamma_{a}-\gamma V_{a}, \\
& \gamma^{a}:=T_{\gamma^{\circ}} \sigma^{a}=\gamma^{-1} \sigma^{a}, \\
& \gamma_{q^{k}}:=T_{\gamma^{\circ}} q^{k}=\gamma^{-(-1)^{2 s_{k s}} k_{k} / 2} q^{k}, \\
& { }^{\gamma} p_{k}:=T_{\gamma^{\circ}} p_{k}=\gamma^{(-1)^{2 s_{k s} / 2}} p_{k} .
\end{aligned}
$$

The standard Thiemann transform would be attained with $\gamma=-i$, except for the fact that our extended transform not only acts on fields but also on coupling constants. Actually, for $\gamma=-i, T_{\gamma}$ reproduces the modification of the Thiemann transform proposed in Ref. [5], which has a nontrivial effect on $\kappa[7]$. We have

$$
T_{\gamma}^{\circ} \kappa=\gamma^{-D(\kappa) / 2} \kappa
$$

From the above equations, we see that our extended Thiemann transform preserves the canonical Poisson brackets and canonically implements the change from one AshtekarBarbero connection to another. Note also that $T_{\gamma}$ leaves invariant the shift and densitized lapse,

$$
T_{\gamma}^{\circ} N=N, \quad T_{\gamma} \circ N^{a}=N^{a} .
$$

Therefore,

$$
\begin{aligned}
\gamma d s^{2}:= & T_{\gamma} \circ d s^{2}=-\sigma^{2} \gamma^{-3} N^{2} d t^{2}+\gamma^{-1} h_{a b}\left(d x^{a}+N^{a} d t\right)\left(d x^{b}\right. \\
& \left.+N^{b} d t\right)
\end{aligned}
$$

i.e., it amounts to a constant scale transformation of the line element plus a change of lapse.

Introducing an index $\alpha$ to denote the different fields present in the theory (gravitational, scalar, fermionic, and Yang-Mills fields), the extended Thiemann transform can be further generalized to

$$
T_{\left\{\gamma_{\alpha}\right\}}=\prod_{\alpha} T_{\gamma_{\alpha}}^{\alpha}
$$

where $T_{\gamma_{\alpha}}^{\alpha}$ is the restriction of $T_{\gamma_{\alpha}}$ to the field $\alpha$ (viewed as a canonical pair of phase-space variables) and its associated coupling constant $\kappa_{\alpha}$. In other words, we may allow each field $\alpha$ to transform according to its own parameter $\gamma_{\alpha}$.
It is easy to check that, when acting on phase-space variables, the transformation $T_{\left\{\gamma_{\alpha}\right\}}$ is generated via Poisson brackets by [7]

$$
\mathcal{T}_{\left\{\gamma_{\alpha}\right\}}=\frac{i}{2} \sum_{\alpha} \ln \gamma_{\alpha} D\left(q^{\alpha}\right) \int d^{3} x p_{\alpha} q^{\alpha},
$$

where $\left\{q^{\alpha}\right\}$ denotes the set of variables $\left\{-\sqrt{2} \sigma^{a}, q^{k}\right\}$, their canonically conjugate variables $\left\{V_{a}, p_{k}\right\}$ are denoted as $\left\{p_{\alpha}\right\}$, and traces over $S U(2)$ and Yang-Mills indices are implicitly assumed. The generator of the standard Thiemann transform is obtained for $\gamma_{\alpha}=-i, \forall \alpha$ [7].

As mentioned above, the connections $\gamma_{a}$ are still complex in the presence of fermions, because $V_{a}$ then has a non-vanishing imaginary contribution. More precisely, let us use the Pauli matrices $\tau_{A}^{j}{ }^{B}$ (with $j=1,2,3$ ) to express the Ashtekar-Barbero variables in the form [2]

$$
{ }^{\gamma} \sigma^{a}=-\frac{i}{\sqrt{2}}{ }^{\gamma} \sigma_{j}^{a} \tau^{j}, \quad \gamma_{a}=-\frac{i}{2}{ }^{\gamma} a_{a}^{j} \tau^{j},
$$

where $\sigma_{j}^{a}=\gamma^{\gamma} \sigma_{j}^{a}$ is the densitized triad. It turns out that the imaginary part of $V_{a}^{j}=i \operatorname{tr}\left(V_{a} \tau^{j}\right)$ can be taken equal to $-i \sigma_{a}^{j} \operatorname{tr}(y) / 4$ [7], which differs from zero when the system includes fermions. In that case, one can still recover a real connection by simply replacing $\gamma_{a}^{j}$ with its real part, namely, with

$$
{ }^{\gamma} A_{a}^{j}=\gamma_{a}^{j}+\frac{{ }^{\gamma} \sigma_{a}^{j}}{4} \operatorname{tr}\left({ }^{\gamma} y\right) .
$$

Here, we have employed that $y=\gamma y$ and $\gamma \sigma_{a}=\gamma_{\sigma_{a}}$. More importantly, it is not difficult to check that the above change of connections can indeed be promoted to a canonical transformation by introducing the following new set of fermionic variables [7]:

$$
\left\{{ }^{\gamma} \xi^{\prime},{ }^{\gamma} \rho^{\prime},{ }^{\gamma} \bar{\eta}^{\prime},{ }^{\gamma} \omega^{\prime}\right\}=\left\{\sqrt{\gamma_{\sigma}}{ }^{\gamma} \xi,{ }^{\gamma} \rho / \sqrt{\gamma} \sigma, \sqrt{\gamma} \sigma^{\gamma} \bar{\eta},{ }^{\gamma} \omega / \sqrt{\gamma} \sigma\right\},
$$

with ${ }^{\gamma} \sigma=\sqrt{\operatorname{det}\left({ }^{\gamma} \sigma_{j}^{a}\right)}$. In this way, one attains the desired description of the gravitational field in terms of a real $S U(2)$ connection while preserving the canonical structure on phase space. Finally, note that from the definition of the new fermionic fields and the connection ${ }^{\gamma} A_{a}$, the extended Thiemann transform continues to map canonical variables with $\gamma=1$ to their counterpart with a generic value of the positive parameter $\gamma$.

\section{KINEMATICS AND IMMIRZI AMBIGUITY}

The kinematical constraints (2.3) can be written in terms of the real connections ${ }^{\gamma} A_{a}$ and the rest of the new canonical variables introduced in the previous section. After some calculations that involve the compatibility between the spin connection and the soldering form, the Bianchi identities, and the relations between Pauli matrices $\left(\tau^{j} \tau^{k}\right)_{A}{ }^{B}$ $=i \epsilon^{j k l} \tau_{A}^{l{ }^{B}}+\delta^{j k} \delta_{A}{ }^{B}[2]$, the constraints become 


$$
\begin{aligned}
\mathrm{G} & =\gamma_{g}{ }^{-1 \gamma} \mathrm{D}_{a}{ }^{\gamma} \mathbb{E}^{a}:=\gamma_{\mathrm{G}} \\
\mathcal{G}_{A B} & =-\sqrt{2}{ }^{\gamma} \mathcal{D}_{a}{ }^{\gamma} \sigma_{A B}^{a}-\gamma^{\gamma}{ }_{(A B)}^{\prime}:={ }^{\gamma} \mathcal{G}_{A B} \\
\mathcal{V}_{a} & =\gamma^{\gamma} \mathcal{V}_{a}+\frac{i}{4 \sqrt{2} \gamma} \operatorname{tr}\left({ }^{\gamma} y^{\prime}\right) \operatorname{tr}\left({ }^{\gamma} \mathcal{G}{ }^{\gamma} \sigma_{a}\right)+\frac{i}{\gamma} \operatorname{tr}\left[{ }^{\gamma} \mathcal{G}\left(\Gamma_{a}-{ }^{\gamma} A_{a}\right)\right]
\end{aligned}
$$

where ${ }^{\gamma} \mathcal{D}_{a}$ is the derivative operator associated with the connection ${ }^{\gamma} A_{a},{ }^{\gamma} F_{a b}$ is its curvature, and

$$
\begin{aligned}
{ }^{\gamma} \mathcal{V}_{a}:= & \sqrt{2} \operatorname{tr}\left({ }^{\gamma} \sigma^{b \gamma} F_{a b}\right)-\frac{1}{2}\left({ }^{\gamma} \rho_{A}^{\prime}{ }^{\gamma} \mathcal{D}_{a}{ }^{\gamma} \xi^{\prime A}+{ }^{\gamma} \omega_{A}^{\prime}{ }^{\gamma} \mathcal{D}_{a}{ }^{\gamma} \bar{\eta}^{\prime A}\right) \\
& +\frac{1}{2}\left({ }^{\gamma} \mathcal{D}_{a}{ }^{\gamma} \rho_{A}^{\prime}{ }^{\gamma} \xi^{\prime A}+{ }^{\gamma} \mathcal{D}_{a}{ }^{\gamma} \omega_{A}^{\prime}{ }^{\gamma} \bar{\eta}^{\prime A}\right)-{ }^{\gamma} \pi_{\phi} \partial_{a}{ }^{\gamma} \phi \\
& -\frac{1}{2} \operatorname{tr}\left({ }^{\gamma} \mathbb{E}^{b \gamma} \mathbb{B}_{a b}\right)-\operatorname{tr}\left[{ }^{\gamma} \mathcal{G}\left(\Gamma_{a}-{ }^{\gamma} A_{a}\right)\right]
\end{aligned}
$$

In addition, $\gamma^{\prime} y_{(A B)}^{\prime}$ is the counterpart of expression (2.2) for the new fermionic variables, and ${ }^{\gamma} \mathbb{D}_{a}{ }^{\gamma} \mathbb{E}^{a}$ and ${ }^{\gamma} \mathbb{B}_{a b}$ stand for the result of evaluating Eqs. (2.4) and (2.5), respectively, at the scaled Yang-Mills variables $\left({ }^{\gamma} \mathrm{A}_{a},{ }^{\gamma} \mathbb{E}^{a}\right)$ employing the new coupling constant $\gamma_{g}:=C_{\gamma^{-1 / 2}}{ }^{\circ}$.

We hence see that the physical constraints of Lorentzian general relativity $\left\{\chi_{l}\right\}=\left\{G, \mathcal{G}, \mathcal{V}_{a}\right\}$ are equivalent to the new constraints $\left\{{ }^{\gamma} \chi_{l}\right\}=\left\{{ }^{\gamma}{ }_{G},{ }^{\gamma} \mathcal{G}^{\gamma}{ }^{\gamma} \mathcal{V}_{a}\right\}$. We can therefore use the latter as the kinematical constraints in the real connection formulation. Furthermore, note that the functional dependence of ${ }^{\gamma} \chi_{l}$ on the corresponding set of canonical variables, specified by the parameter $\gamma$, is the same for all values of $\gamma$ provided that the Yang-Mills coupling constant is also scaled with this parameter (according to the definition of $\gamma_{g}$ ). Actually, this property continues to hold even when we allow the parameter $\gamma$ to take different values for each of the fields that are present in the system, namely, when the canonical variables and coupling constants denoted with the index $\gamma$ are in fact those obtained with the set of parameters $\left\{\gamma_{\alpha}\right\}$ introduced in Eq. (3.11).

It is now straightforward to check that the extended Thiemann transform $T_{\left\{\gamma_{\alpha}\right\}}$ leaves the kinematical structure invariant. We have already seen that the transform preserves the canonical Poisson brackets. Then, we only have to show that it does not alter the kinematical constraints. By construction, $T_{\left\{\gamma_{\alpha}\right\}}$ maps the canonical set of variables (and the coupling constants) with $\gamma=1$ to those with parameters $\left\{\gamma_{\alpha}\right\}$; so,

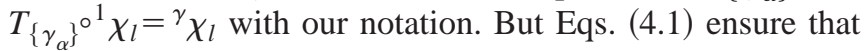
$\left\{{ }^{\gamma} \chi_{l}\right\}$ is equivalent to $\left\{{ }^{1} \chi_{l}\right\}$. The set of constraints $\left\{{ }^{1} \chi_{l}\right\}$ is thus invariant under the extended Thiemann transform. As a consequence, we can identify the symplectomorphism $T_{\left\{\gamma_{\alpha}\right\}}$ with the identity transformation at the kinematical level.

Since, $T_{\left\{\gamma_{\alpha}\right\}} \equiv 1$ as far as the kinematical structure is concerned, the action of the transforms $R_{\left\{\gamma_{\alpha}\right\}}$ and $C_{\left\{\gamma_{\alpha}^{1 / 2}\right\}}$ can be regarded as equivalent at this level. It is worth noting that the latter of these transforms amounts to a constant change of scale if (and only if) $\gamma_{\alpha}=\gamma, \forall \alpha$. To see this point clearly, it is convenient to introduce a formalism in which all fields and coupling constants (that we denote generically by the symbol
$X)$ are dimensionless. This can be accomplished by introducing the universal length scale $l_{*}$ and defining ${ }^{*} X$ $:=l_{*}^{-D(X)} X$, so that

$$
C_{\gamma_{\alpha}^{-1 / 2} \circ *} X=* X \text { and } C_{\gamma_{\alpha}^{-1 / 2} \circ l_{*}}=\gamma_{\alpha}^{-1 / 2} l_{*}
$$

Obviously, the scaling transformations of $l_{*}$ for each kind of field $\alpha$ are incompatible unless all the parameters $\gamma_{\alpha}$ coincide. Then, in the case $\gamma_{\alpha}=\gamma \forall \alpha$, we see that the action of the extended Wick transform can indeed be interpreted as a constant scale transformation if we restrict ourselves to kinematical considerations:

$$
R_{\gamma} \equiv C_{\gamma^{1 / 2}}
$$

The main reason underlying this result is that both the constraints ${ }^{1} \chi_{l}$ and the fundamental non-vanishing Poisson brackets have the same dimension (namely, 2), as well as the same degree of homogeneity (namely, 1) in the variables

$$
\left\{P_{\alpha}\right\}:=\left\{\Gamma_{a}-{ }^{1} A_{a}, \pi_{\phi},{ }^{1} \rho^{\prime},{ }^{1} \omega^{\prime}, \mathbb{E}^{a}\right\},
$$

up to terms that vanish because of the Bianchi identities or the compatibility of the triad with the spin connection. Notice that the extended Wick transform $R_{\gamma}$ multiplies each of the elements in $\left\{P_{\alpha}\right\}$ by a factor of $\gamma$ while leaving invariant their canonically conjugate variables

$$
\left\{Q^{\alpha}\right\}:=\left\{-\sqrt{2} \sigma^{a}, \phi,{ }^{1} \xi^{\prime},{ }^{1} \bar{\eta}^{\prime}, \mathrm{A}_{a}\right\} .
$$

We now want to discuss the implications of the equivalence (4.4) for the Immirzi ambiguity that arises in loop quantum gravity at the kinematical level. With this aim, let us consider an (abstract) operator $\vartheta(\sigma)$ constructed from the soldering form, its dimension being $D(\vartheta)$. We assume that this operator is an observable, at least from a kinematical point of view. Let us call $\mathrm{Sp}[\vartheta(\sigma)]$ and $\operatorname{Sp}_{\gamma}[\vartheta(\sigma)]$ its spectra in the loop representations based, respectively, on the real connections ${ }^{1} A_{a}$ and ${ }^{\gamma} A_{a}$, adopting in both cases as configuration variables for the matter fields the elements of $\left\{Q^{\alpha}\right\}$ other than the soldering form. Since $R_{\gamma}{ }^{\circ} Q^{\alpha}=Q^{\alpha}$ and $R_{\gamma}{ }^{\circ} A_{a}={ }^{\gamma} A_{a}$, the two representations are related via the extended Wick transform. Using in our kinematical analysis that $R_{\gamma} \equiv C_{\gamma^{1 / 2}}$, one concludes that the spectrum $\operatorname{Sp}_{\gamma}[\vartheta(\sigma)]$ is the image of $\operatorname{Sp}[\vartheta(\sigma)]$ under a constant scale transformation: namely,

$$
\operatorname{Sp}_{\gamma}[\vartheta(\sigma)]=\operatorname{Sp}\left[C_{\gamma^{1 / 2}} \circ \vartheta(\sigma)\right]=\gamma^{D(\vartheta) / 2} \operatorname{Sp}[\vartheta(\sigma)]
$$

This spectrum is obviously different from $\operatorname{Sp}[\vartheta(\sigma)]$ if it contains a discrete component, as happens for instance for the area operator [26].

The discrepancy between the spectra of geometric operators in the loop representations obtained with different connections ${ }^{\gamma} A_{a}$ is called the Immirzi ambiguity [10]. As we have seen, the ambiguity existing in vacuo persists also in the presence of matter fields. But much more importantly, our discussion shows that, owing to the relation $R_{\gamma} \equiv C_{\gamma^{1 / 2}}$, 
the Immirzi ambiguity can be considered equivalent to a constant scale transformation at the kinematical level.

We should note that the presence of matter has introduced a subtle modification in our analysis with respect to the pure gravitational situation. As a result of a direct extrapolation of the conclusions for vacuum gravity, the Immirzi ambiguity is usually thought to arise in loop representations that are related by a transform like $R_{\gamma}$, but restricted to act only on the gravitational field. With the notation of Sec. III, this would correspond to the replacement of $\gamma$ with a collection of parameters $\left\{\gamma_{\alpha}\right\}$ (one for each field) such that they all equal the unity except for the gravitational component. The remarkable point is that the same Immirzi ambiguity appears when the extended Wick transform acts on all fields with identical value of $\gamma$. Moreover, as we have proved, the transform can be consistently interpreted in this case, from a kinematical point of view, as a constant scale transformation that equally affects all fields and parameters, not only those associated with the gravitational sector.

Finally, it should be stressed that the equivalence between the extended Wick transform (whose implementation results in the Immirzi ambiguity) and a constant scale transformation is only valid for kinematical considerations. Indeed, as shown in the Appendix, the scalar constraint is not invariant under the action of $T_{\gamma}$. Therefore, the equivalence does not hold dynamically and, consequently, the Immirzi ambiguity cannot be associated with the multiplication of the classical action by a constant (removable by means of a change of scale) when dynamics is taken into account $[13,17]$.

\section{BLACK HOLE ENTROPY}

A particular example of a geometric operator with a discrete spectrum is that representing the area $\mathcal{A}$ of an isolated horizon in loop quantum gravity $[22,26]$. Since the dimension of the area operator is 2, Eq. (4.7) gives the relation between the eigenvalues $\mathcal{A}(J)$ and $\mathcal{A}_{\gamma}(J)$ in the respective loop representations with connections ${ }^{1} A_{a}$ and ${ }^{\gamma} A_{a}$ :

$$
\mathcal{A}_{\gamma}(J)=\gamma \mathcal{A}(J) .
$$

Here, the symbol $J$ labels the different eigenvalues of the spectrum.

The explicit form of the area spectrum for an isolated horizon, which is deduced from purely kinematical considerations, is one of the keystones in the calculation of the statistical entropy $S$ of a black hole, obtained in the Ashtekar approach by counting degrees of freedom in the Hilbert space of loop quantum gravity. An important point in this deduction of the black hole entropy is that, since the full theory of quantum gravity is not known, the calculation is carried out by studying only the sector of isolated horizons with constant area $\mathcal{A}$. This sector is quantized in a loop representation with a certain Immirzi parameter $\gamma$. In the limit of a large horizon area, the resulting entropy is $[21,22]$

$$
S=\frac{\gamma_{0} \mathcal{A}}{4 \gamma l_{*}^{2}}, \quad \text { with } \quad \gamma_{0}=\frac{\ln 2}{\pi \sqrt{3}} .
$$

To recover the Bekenstein-Hawking formula $S=\mathcal{A} /\left(4 l_{p}^{2}\right)$ (where $l_{p}$ is the Planck length of low-energy physics), it is then argued that the Immirzi parameter must be fixed so that $\gamma l_{*}^{2}=\gamma_{0} l_{p}^{2}$. In particular, if $l_{*}$ and $l_{p}$ coincide, $\gamma$ must be chosen equal to $\gamma_{0}$ in order to reach an acceptable semiclassical prediction.

There is however a potential loophole in the above line of reasoning that had remained unperceived so far. Namely, since the calculation of the black hole entropy is performed for a fixed value of the horizon area and with a certain choice of the Immirzi parameter, there seems to be no obstacle to select $\gamma$ in terms of $\mathcal{A}$, so that $\gamma$ becomes in practice a function of this quantity. Obviously, this would spoil the linear relation between the entropy and the area. A way out of this problem consists in proving that the appearance of the Immirzi parameter in the entropy formula amounts in fact to a change of scale. As we will show, imposing that the Planck scale be unique for all the observers that carry out the measurements will then eliminate the postulated dependence of $\gamma$ on the area.

Note that, to attain our goal, we only have to demonstrate that the Immirzi parameter can be absorbed through a constant scaling in all the steps where the dynamical structure enters the calculation of the entropy. For all other considerations we simply have to apply the results of Sec. IV, where we proved the kinematical equivalence of the Immirzi ambiguity with a change of scale. Actually, the dynamical structure appears in the entropy calculation only through the isolated-horizon boundary conditions. These conditions code dynamical information and intervene in the analysis inasmuch as they are utilized to prove the following results $[22,23]$.

(i) The surface terms of the action, necessary for a wellposed variational problem, correspond to a Chern-Simons theory whose $U(1)$ connection is $W_{a}=\operatorname{tr}\left(\underline{\Gamma}_{a} r\right)$. The underline denotes the pull-back to the spatial sections of the horizon, which are topologically $S^{2}$, and $r=-i r_{j} \tau^{j} / \sqrt{2}$ is a fixed smooth function from the sphere to the Lie algebra of $S U(2)$ with $\operatorname{tr}\left(r^{2}\right)=-1$. We note that both $r$ and the spin connection $\Gamma_{a}$ are invariant under constant scale transformations.

(ii) The level $k$ of this $U(1)$ Chern-Simons theory is $k=\mathcal{A} /\left(4 \pi \gamma l_{*}^{2}\right)$, where $\mathcal{A}$ is the constant area of the horizon. This level determines the overall factor in the Chern-Simons contribution to the symplectic structure. Notice that $\gamma$ appears in $k$ exactly as a scaling of the universal length $l_{*}$.

(iii) The curvature of the Chern-Simons connection is $\operatorname{tr}\left({ }^{\gamma} \Sigma_{a b} r\right) /\left(2 k l_{*}^{2}\right)$. Here, $\Sigma_{a b}:=\eta_{a b c} \sigma^{c}$ and $\eta_{a b c}$ is the LeviCività form-density. Since ${ }^{\gamma} \Sigma_{a b}=\gamma^{-1} \Sigma_{a b}$, we see that the Immirzi parameter appears again as a constant scaling of $l_{*}$.

Remarkably, the isolated-horizon boundary conditions ensure in addition that there do not exist non-trivial Hamiltonian gauge transformations at the horizon, so that we do not have to impose the scalar constraint on it. This means that the dynamical aspects of the matter content do not affect the physics at the horizon and, as a consequence, the specific choice of the Immirzi parameter for the matter fields does not affect the entropy calculation. 
The only issue, purely non-dynamical, remaining in the calculation of the entropy is counting the physical states whose area eigenvalue $\mathcal{A}_{\gamma}(J)$ (in the loop representation based on ${ }^{\gamma} A_{a}$ ) lies within the interval

$$
\frac{\mathcal{A}}{\gamma l_{*}^{2}}-\delta \leqslant \frac{\mathcal{A}_{\gamma}(J)}{\gamma l_{*}^{2}} \leqslant \frac{\mathcal{A}}{\gamma l_{*}^{2}}+\delta .
$$

The counting is made for large $\mathcal{A} /\left(\gamma l_{*}^{2}\right)$ and assuming that $\delta>4 \pi \sqrt{3}$ (to ensure the existence of at least one eigenvalue that corresponds to an even number of spin insertions). Following the steps in [22], one then obtains the entropy formula (5.2).

As we have already argued, the Immirzi ambiguity is kinematically equivalent to a constant scale transformation. We have also seen that all the dynamical arguments involved in the entropy calculation indicate that $\gamma$ appears as a constant scaling of the universal length $l_{*}$, even though the scalar constraint and hence the dynamics in general do not support such interpretation. Therefore, we can conclude that the appearance of the Immirzi ambiguity in the entropy formula is equivalent to a scaling of $l_{*}$.

In our discussion, there seem to exist two length scales which, for the time being, have been treated as independent [19]. One of them would be the fundamental length scale $l_{*}$, which appears in front of the action and determines the Poisson bracket structure $[7,20]$, and hence the strength of the quantum gravitational effects. The other would be a lowenergy length scale, which would characterize the lowenergy behavior of quantum gravity, and whose square would provide the quantum of area. Let us call this length scale the Planck length and define it as

$$
l_{p}=l_{*} \sqrt{\gamma / \gamma_{0}},
$$

so that

$$
S=\frac{\mathcal{A}}{4 l_{p}^{2}} .
$$

Our definition of $l_{p}$ is feasible because, as we have shown, the Immirzi ambiguity amounts to a change of scale, at least as far as the area spectrum and the entropy formula are concerned. From this point of view, fixing $\gamma$ is equivalent to fixing the effective value of the low-energy Planck length $l_{p}$ in terms of the fundamental length $l_{*}$.

Let us remember that, because the whole entropy analysis has been performed under the assumption that the area of the isolated horizon is fixed and given a priori, the Immirzi parameter might in principle be made dependent on $\mathcal{A}$, thus spoiling the linearity of the relation between entropy and area. Fortunately, the possible effects of this potential dependence of $\gamma$ on the area can now be eliminated on the basis of a physical requirement: when comparing low-energy and large-horizon physics for different horizons, the comparison must be carried out by observers that assign the same value to the Planck length $l_{p}$. Since $l_{*}$ is a universal constant and $\gamma_{0}$ is just a numerical factor, this fixes the value of $\gamma$ to be the same for all observers and, of course, independent of the area.

\section{SUMMARY AND DISCUSSION}

In this paper, we have shown that the Immirzi ambiguity can be described at a kinematical level in terms of constant scale transformations. With this aim, we have considered the Ashtekar-Barbero formulation of general relativity coupled with fermions, a scalar field, and a Yang-Mills field. In this framework, the Immirzi ambiguity appears when one calculates the spectra of geometric operators using loop representations that are based on different real connections for the gravitational field. We have shown that these representations can be related via an extended Wick transform that, in addition to introducing the Immirzi parameter in the gravitational connection, has also the effect of multiplying the matter momenta by the same parameter. This extended Wick transform admits a geometric interpretation as a scaling of the lapse function, and we have proved that it can be completed with a constant scale transformation to reach a symplectomorphism. In a sense, the constructed symplectomorphism provides an extension of the Thiemann transform that maps the Lorentzian to the Euclidean formulation of Ashtekar gravity.

Such an extended Thiemann transform has been shown to preserve the kinematical constraints of the system, so that it can be viewed as equivalent to the unit transform as far as one disregards the dynamical evolution. Based on this fact, we have argued that the Immirzi ambiguity in loop quantum gravity can be understood in terms of a constant scale transformation for all kinematical considerations. Indeed, the physical spectra of geometric operators in loop quantum gravity are affected by the Immirzi parameter in a way which appropriately depends on the dimension of the operators. The corresponding scale transformation implies a change of conformal frame that can be considered responsible for the quantum ambiguity.

The scalar constraint, on the other hand, is not invariant under the extended Thiemann transform, and hence the Immirzi ambiguity cannot be absorbed dynamically into a change of scale. This can also be rephrased by saying that the four-dimensional line elements obtained with a constant scale transformation and with a constant scaling of the lapse do not lead to dynamically equivalent theories. The breakdown of this equivalence with respect to the kinematical situation makes us suspicious of the special role played by time in the Ashtekar-Barbero formulation, which can be traced back to the time gauge fixing that is introduced in such a formalism and the consequent loss of a genuine spacetime interpretation for the gravitational connection [10-12].

The Immirzi ambiguity affects one of the most outstanding predictions of loop quantum gravity, namely, the entropy formula for isolated horizons. The derivation of this formula involves not only kinematical but also dynamical processes. However, the dynamical structure turns out to enter the calculation only coded in the isolated-horizon boundary conditions. It is worth commenting that an analogous conclusion 
has been recently reached by Padmanabhan from a completely independent point of view [27]. He has deduced the entropy of a spherically symmetric spacetime with a horizon by studying the partition function of a canonical ensemble with fixed temperature on that horizon. This analysis does not assume that the spacetimes in the ensemble are solutions to the Einstein equations; moreover, it is seen that the result depends only on the form of the metric near the boundary supplied by the horizon.

Returning to our study of the loop approach, we have shown that, whenever the Immirzi parameter appears in the calculation of the black hole entropy through the conditions on the horizon, remarkably, it behaves in fact as though it came from a constant scale transformation. The remaining arguments that lead to the entropy formula are strictly kinematical. It then follows from our discussion that the Immirzi ambiguity in the relation between entropy and area can be understood as a conformal ambiguity in the length scale employed to measure large horizon areas and low-energy processes in general. This is important because the entropy calculation is performed for isolated horizons with a constant area that is given a priori. Therefore, the choice of Immirzi parameter in the loop quantization might in principle be made dependent on this area, and this would ruin the possibility of deducing the Bekenstein-Hawking formula. However, this potential dependence on the area of the Immirzi parameter disappears if we insist that the results for different large isolated horizons be compared by observers which agree on the value of the length scale that controls the semiclassical gravitational effects from the low-energy point of view, i.e., Planck length, because the Immirzi parameter can be absorbed into this length scale.

\section{ACKNOWLEDGMENTS}

G.A.M.M. is very thankful to J.F. Barbero G. for enlightening discussions. This work was supported by funds provided by the Spanish Ministry of Science and Technology under the Research Project No. BFM2001-0213.

\section{APPENDIX A: SCALAR CONSTRAINT}

In this appendix, we present the expressions of the scalar constraint in terms of the Ashtekar connection and of the real connections ${ }^{\gamma} A_{a}$. In the Ashtekar formulation of Lorentzian gravity with matter fields, the scalar constraint is $[2,3,7]$

$$
\begin{aligned}
\mathcal{S}= & -\operatorname{tr}\left(\sigma^{a} \sigma^{b} \mathcal{F}_{a b}\right)+i m\left(\sigma^{2} \xi^{A} \bar{\eta}_{A}-\rho^{A} \omega_{A}\right) \\
& +i \sqrt{2} \sigma_{A}^{a}{ }_{A}^{B}\left(\rho_{B} \breve{\mathcal{D}}_{a} \xi^{A}+\omega_{B} \breve{\mathcal{D}}_{a} \bar{\eta}^{A}\right)+\sigma^{2} \Lambda+\frac{\pi_{\phi}^{2}}{16 \pi} \\
& +4 \pi \sigma^{2} \mu^{2} \phi^{2}-4 \pi \operatorname{tr}\left(\sigma^{a} \sigma^{b}\right) \partial_{a} \phi \partial_{b} \phi \\
& +\frac{1}{8 \sigma^{2}} \operatorname{tr}\left(\sigma^{a} \sigma^{c}\right) \operatorname{tr}\left(\sigma^{b} \sigma^{d}\right) \operatorname{tr}\left(\mathbb{E}_{a b} \mathbb{E}_{c d}+\mathbb{B}_{a b} \mathbb{B}_{c d}\right),
\end{aligned}
$$

where we have employed the notation of Sec. II, $m$ and $\mu$ denote the masses of the fermionic and scalar fields, $\Lambda$ is the cosmological constant, and $\mathbb{E}_{a b}=\eta_{a b c} \mathbb{E}^{c}$, with $\eta_{a b c}$ being the Levi-Cività form-density.

This constraint leads to the Einstein-Cartan theory, which is quartic in the fermionic variables. Nevertheless, one can attain the Einstein-Dirac theory, quadratic in fermionic fields, by simply adding to $\mathcal{S}$ the term [2,7]

$$
\mathcal{S}_{\mathrm{f}}=-\frac{3}{16}\left(y_{A}{ }^{A} y_{B}{ }^{B}+y_{A B} y^{A B}+y_{A B} y^{B A}\right) .
$$

In terms of the real connection ${ }^{\gamma} A_{a}$, the scaled soldering form ${ }^{\gamma} \sigma^{a}$, and the canonical set of matter variables specified by the parameter $\gamma$ (here, we concentrate on the case of interest $\gamma_{\alpha}=\gamma \forall \alpha$ ), the scalar constraint can be written as follows:

$$
\begin{aligned}
\mathcal{S}= & \gamma^{2}{ }^{\gamma} \mathcal{S}+\frac{i}{\sqrt{2}} \gamma(\gamma-1)^{\gamma} Y-\left(\gamma^{2}-1\right)^{\gamma} Z \\
& -\frac{i}{\sqrt{2}} \gamma D_{a} \operatorname{tr}\left({ }^{\gamma} \mathcal{G}^{\gamma} \sigma^{a}\right),
\end{aligned}
$$

where $D_{a}$ is the derivative operator compatible with the triad, obtained with the spin connection $\Gamma_{a}$, and

$$
\begin{aligned}
& { }^{\gamma} \mathcal{S}:=-\operatorname{tr}\left({ }^{\gamma} \sigma^{a} \gamma^{\gamma} \sigma^{b}{ }^{\gamma} F_{a b}\right)+\operatorname{tr}\left({ }^{\gamma} \sigma^{a}{ }^{\gamma} \sigma^{b}\left[\Gamma_{a}-{ }^{\gamma} A_{a}, \Gamma_{b}-{ }^{\gamma} A_{b}\right]\right) \\
& +{ }^{\gamma} \sigma^{2}{ }^{\gamma} \Lambda+i{ }^{\gamma} m{ }^{\gamma} \sigma{ }^{\gamma} \xi^{\prime A \gamma} \bar{\eta}_{A}^{\prime}+\frac{1}{\sqrt{2}} \operatorname{tr}\left({ }^{\gamma} \sigma D_{a}{ }^{\gamma} y^{\prime}\right) \\
& +4 \pi^{\gamma} \sigma^{2}{ }^{\gamma} \mu^{2}{ }^{\gamma} \phi^{2}-4 \pi \operatorname{tr}\left({ }^{\gamma} \sigma^{a}{ }^{\gamma} \sigma^{b}\right) \partial_{a}{ }^{\gamma} \phi \partial_{b}{ }^{\gamma} \phi \\
& +\frac{1}{8^{\gamma} \sigma^{2}} \operatorname{tr}\left({ }^{\gamma} \sigma^{a}{ }^{\gamma} \sigma^{c}\right) \operatorname{tr}\left({ }^{\gamma} \sigma^{b}{ }^{\gamma} \sigma^{d}\right) \operatorname{tr}\left({ }^{\gamma} \mathbb{B}_{a b}{ }^{\gamma} B_{c d}\right) \\
& +{ }^{\gamma} Z-\frac{i}{\sqrt{2}}{ }^{\gamma} Y+\frac{1}{\sqrt{2}} D_{a} \operatorname{tr}\left({ }^{\gamma} \mathcal{G}{ }^{\gamma} \sigma^{a}\right) \\
& { }^{\gamma} Y:=\operatorname{tr}\left({ } ^ { \gamma } \sigma ^ { a } \left\{D_{a}^{\gamma} \rho^{\prime \gamma} \xi^{\prime}+D_{a}^{\gamma} \omega^{\prime \gamma} \bar{\eta}^{\prime}-{ }^{\gamma} \rho^{\prime} D_{a}^{\gamma} \xi^{\prime}\right.\right. \\
& \left.\left.-\gamma \omega^{\prime} D_{a}^{\gamma} \bar{\eta}^{\prime}\right\}\right) \\
& { }^{\gamma} Z:=\operatorname{tr}\left({ }^{\gamma} \sigma^{a}{ }^{\gamma} \sigma^{b}\left[\Gamma_{a}-{ }^{\gamma} A_{a}, \Gamma_{b}-{ }^{\gamma} A_{b}\right]\right)+\frac{3}{16}\left[\operatorname{tr}\left(\gamma^{\gamma}{ }^{\prime}\right)\right]^{2} \\
& -i^{\gamma} m{ }^{\gamma} \sigma{ }^{\gamma} \rho^{\prime A} \gamma_{\omega_{A}^{\prime}}-\frac{1}{\sqrt{2}} \operatorname{tr}\left({ }^{\gamma} y^{\prime} \tau^{k}\right) \operatorname{tr}\left({ }^{\gamma} \sigma^{a} \tau^{k}\left\{\Gamma_{a}-{ }^{\gamma} A_{a}\right\}\right) \\
& +\frac{{ }^{\gamma} \pi_{\phi}^{2}}{16 \pi}+\frac{1}{8^{\gamma} \sigma^{2}} \operatorname{tr}\left({ }^{\gamma} \sigma^{a}{ }^{\gamma} \sigma^{c}\right) \operatorname{tr}\left({ }^{\gamma} \sigma^{b}{ }^{\gamma} \sigma^{d}\right) \operatorname{tr}\left({ }^{\gamma} \mathbb{E}_{a b}{ }^{\gamma} \mathbb{E}_{c d}\right) .
\end{aligned}
$$

We have used the notation introduced in Secs. III and IV, and the scaled coupling constants are $\gamma_{\kappa:=} \gamma^{-D(\kappa) / 2} \kappa . D(\kappa)$ is equal to -1 except for $\Lambda$, whose dimension is -2 .

Taking $\gamma=1$, we see from the above expressions that ${ }^{1} \mathcal{S}=\mathcal{S}$ modulo the gravitational Gauss constraint. We can 
hence use ${ }^{1} \mathcal{S}$ as the scalar constraint in the real connection formulation with $\gamma=1$. On the other hand, recalling that the different sets of canonical phase-space variables and coupling constants parametrized by $\gamma$ are related by the extended Thiemann transform $T_{\gamma}$ and using our definition of ${ }^{\gamma} \mathcal{S}$, we straightforwardly obtain that $T_{\gamma}{ }^{0} \mathcal{S}={ }^{\gamma} \mathcal{S}$. However,
$\gamma_{\mathcal{S}}$ and ${ }^{1} \mathcal{S}$ differ when $\gamma \neq 1$ even modulo the kinematical constraints, as can be easily checked from Eq. (A1). As a consequence, the dynamical structure is not invariant under the extended Thiemann transform. Therefore, the kinematical equivalence $R_{\gamma} \equiv C_{\gamma^{1 / 2}}$ is not maintained when dynamics is taken into account.
[1] A. Ashtekar, Phys. Rev. Lett. 57, 2244 (1986); Phys. Rev. D 36, 1587 (1987).

[2] A. Ashtekar, in Lectures on Non-Perturbative Canonical Gravity, edited by L.Z. Fang and R. Ruffini (World Scientific, Singapore, 1991).

[3] A. Ashtekar, J.D. Romano, and R.S. Tate, Phys. Rev. D 40, 2572 (1989).

[4] T. Thiemann, Class. Quantum Grav. 13, 1383 (1996).

[5] A. Ashtekar, Phys. Rev. D 53, 2865 (1996).

[6] G.A. Mena Marugán, Gravitation Cosmol. 4, 257 (1998).

[7] L.J. Garay and G.A. Mena Marugán, Class. Quantum Grav. 15, 3763 (1998).

[8] J.F. Barbero G., Phys. Rev. D 51, 5507 (1995).

[9] C. Rovelli, Living Rev. Relativ. 1, 1 (1998).

[10] G. Immirzi, Nucl. Phys. B (Proc. Suppl.) 57, 65 (1997); Class. Quantum Grav. 14, L177 (1997).

[11] S. Alexandrov, Class. Quantum Grav. 17, 4255 (2000); Phys. Rev. D 65, 024011 (2001); gr-qc/0201087; S. Alexandrov and D. Vassilevich, Phys. Rev. D 64, 044023 (2001); N. Barros e Sá, Int. J. Mod. Phys. D 10, 261 (2001).

[12] J. Samuel, Class. Quantum Grav. 17, L141 (2000).

[13] C. Rovelli and T. Thiemann, Phys. Rev. D 57, 1009 (1998).

[14] J. Samuel, Phys. Rev. D 64, 048501 (2001).

[15] G.A. Mena Marugán, Class. Quantum Grav. 19, L63 (2002).
[16] A. Corichi and K.V. Krasnov, Phys. Lett. A 13, 1339 (1998).

[17] R. Gambini, O. Obregón, and J. Pullin, Phys. Rev. D 59, 047505 (1999).

[18] M. Montesinos, Class. Quantum Grav. 18, 1847 (2001).

[19] M. Rainer, Gravitation Cosmol. 6, 181 (2000).

[20] K. Krasnov, Class. Quantum Grav. 15, L1 (1998).

[21] A. Ashtekar, J. Baez, A. Corichi, and K. Krasnov, Phys. Rev. Lett. 80, 904 (1998).

[22] A. Ashtekar, J.C. Baez, and K. Krasnov, Adv. Theor. Math. Phys. 4, 1 (2001); T. Thiemann, gr-qc/0110034.

[23] A. Ashtekar, A. Corichi, and K. Krasnov, Adv. Theor. Math. Phys. 3, 419 (2000).

[24] This extended Wick transform should not be mistaken for the Thiemann transform [4,5], which is sometimes referred to as the generalized Wick transform.

[25] Our notation does not make distinctions between fields that depend on the different time coordinates corresponding, respectively, to the original and scaled lapses $N$ and $R_{\gamma}{ }^{\circ} N$. This differs from the notation adopted in Ref. [7], where Euclidean and Lorentzian fields were distinguished for the case of the Wick rotation.

[26] A. Ashtekar, and J. Lewandowsky, Class. Quantum Grav. 14, A55 (1997).

[27] T. Padmanabhan, gr-qc/0202078; gr-qc/0202080. 